\title{
Vigne et vignobles au Saguenay-Lac-Saint-Jean, au Québec : du nouveau dans le paysage ${ }^{1}$
}

\author{
Majella-J. Gauthier
}

RÉSUMÉ. L'activité viticole constitue un phénomène nouveau dans les régions réputées froides comme le Québec, particulièrement dans la région du Saguenay-Lac-Saint-Jean, située au nord de la vallée du SaintLaurent. Le réchauffement climatique, les choix de cépages rustiques, une meilleure détermination des milieux écogéographiques et une ouverture sur la connaissance agronomique des gens constituent le cœur du changement dans le paysage et dans la structure des activités économiques. Si bien qu'aujourd'hui, des dizaines d'entités produisent du raisin et souvent embouteillent du vin aux saveurs originales.

\begin{abstract}
Wine-growing is a new activity in cold regions such as the province of Quebec, and particularly in the Saguenay-LacSaint-Jean, that is located north of the St. Lawrence V alley. Global warming, the choice of rustic grape varieties, a better determination of ecogeographic environments and people opening up to agronomic knowledge have led to the change in the landscape and in the structure of economic activities. So much so that dozens of places now produce grapes and often bottle wine with original flavours.
\end{abstract}

\section{Introduction}

L'installation de la vigne et le développement de vignobles gagnent les régions plus septentrionales de l'Amérique du Nord et le Québec n'y échappe pas. Les conditions climatiques changent et favorisent la présence de milieux agricoles propices à de nouvelles cultures, dont celle de la vigne. La configuration de plusieurs terrains possédant des caractéristiques particulières d'exposition au soleil et de proximité de bonnes surfaces aquatiques joue un rôle déterminant. Si bien que près de 40000 plants de vigne poussent désormais dans la région du Saguenay-Lac-Saint-Jean et qu'une bonne dizaine de vignobles tirent leur épingle du jeu. Ce mouvement n'échappe pas à la notion de nouveauté en agriculture (Ingrand et collab., 2014), car ce mode d'utilisation du sol et d'activité agricole n'existait pas 35 ans auparavant.

Par conséquent, il est intéressant de faire un court rappel historique de l'aventure, de passer en revue les conditions écogéographiques et microclimatiques

a Géographie et professeur émérite, Université du Québec à Chicoutimi dans lesquelles s'effectue la viticulture, de même que de jeter un coup d'œil sur le panorama régional des lieux et des établissements de production de raisin et de vin. Il va de soi que cette nouvelle activité amène des changements heureux dans le paysage, entraîne une diversification économique régionale et se traduit en un souffle de vitalité locale et régionale. Ainsi, la limite nordique de la viticulture s'en trouve repoussée.

\section{Rappel historique}

L'histoire de la vigne au Québec remonte aux premiers explorateurs français. En 1608, Samuel de Champlain a été le précurseur en la matière en effectuant des observations, notamment à Québec. Il faut dire qu'à l'époque, une vigne indigène, la vigne des rivages (Vitis riparia), produisait des raisins sauvages et était naturellement présente au pays. Dans son récit sur le développement des boissons alcoolisées, Ferland (2010) indique que ce sont les Récollets qui ont produit le premier vin au Québec en 1637 avec de la vigne indigène, soit la vigne américaine (Vitis labrusca), tandis que les Sulpiciens 
ont été les premiers à importer un cépage européen, soit la vigne cultivée (Vitis vinifera).

L'histoire de la vigne et du vin au Québec est courte et relativement peu documentée. Sur le plan géographique, Deshaies et Dubois (1993) en effectuent une première esquisse. Puis, leur Guide des vignobles du Québec (Dubois et Deshaies, 1997) trace un tableau éclairant de l'implantation de la vigne. Les auteurs définissent cinq périodes, dont celle allant de 1980 à 1996, durant laquelle il y a ouverture de 90 vignobles, bien souvent au sud du Québec, par exemple à Dunham et ses environs. Depuis ce temps, il y a eu une consolidation de la production, une ouverture au marché, des groupements de producteurs (dont le Conseil des vins du Québec) et une garantie de qualité des vins $\left(\mathrm{IGP}^{2}\right)$. En 2021, on ne compte pas moins de 314 producteurs occupant plus de 1023 hectares.

\section{Le cas du Saguenay-Lac-Saint-Jean}

Dans notre étude géographique réalisée récemment (Gauthier, 2021), il est dit que la viticulture s'est implantée timidement à l'intérieur de la région du Saguenay-Lac-Saint-Jean au cours de la décennie 1980. Or, le vrai coup d'envoi se situe à la fin des années 1990, où plusieurs entités ont mis en terre quelques milliers de plants (voir figure 1).

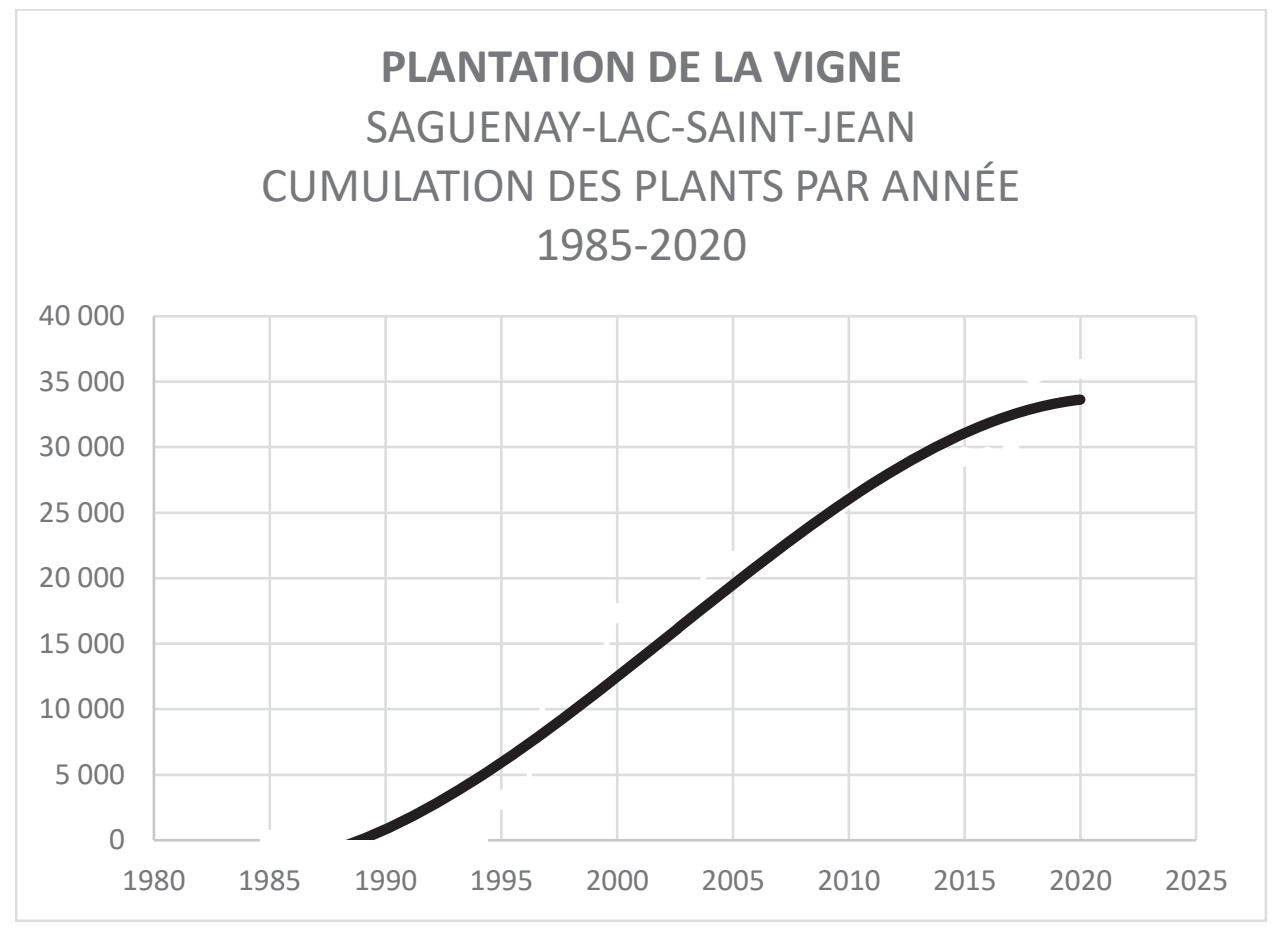

Figure 1 - Tendance dans la plantation de la vigne, Saguenay-Lac-Saint-Jean, 1985-2020

La répartition spatiale des viticulteurs est inégale et épouse un patron relativement clair (voir figure 2). La rive sud et sud-est du lac Saint-Jean, allant de Roberval à Alma, concentre de grandes exploitations, tandis que les basses-terres de chaque côté de la rivière Saguenay rassemblent, dans un corridor allant jusqu'à Petit-Saguenay en aval, des viticulteurs de tailles diverses, dont la plus grande entreprise se situe dans l'arrondissement de Jonquière (Ville Saguenay), avec ses 10000 plants. 


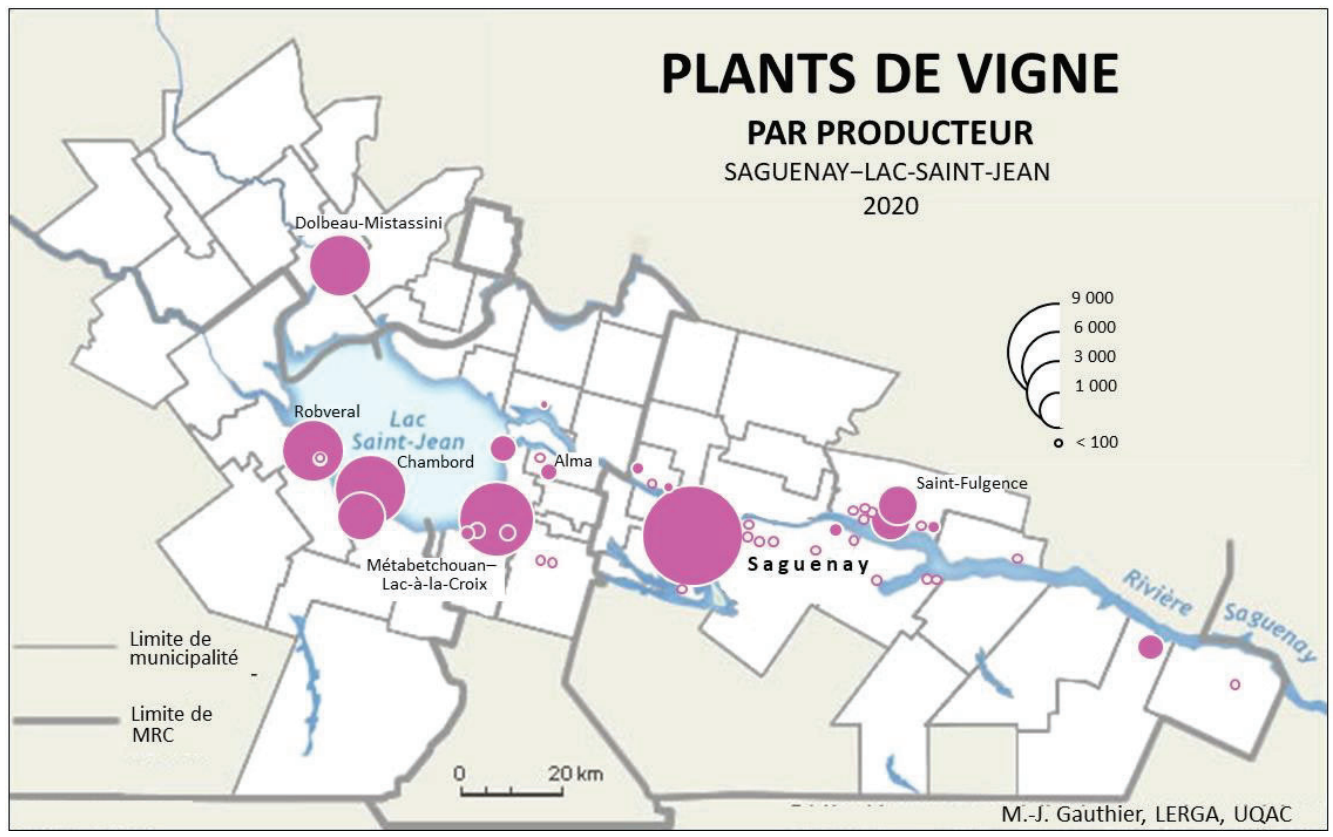

Figure 2 - Plants de vigne par producteur, Saguenay-Lac-Saint-Jean, 2020

La taille des entités n'est pas homogène et se structure ainsi :

- 3 entités : 5 000-10 000 plants;

- 5 entités : 1 000-5 000 plants;

- 10 entités : 100-1 000 plants;

- 27 entités : $<100$ plants.

Les plus grandes entités produisent toutes du vin, mais cela n'est pas exclu chez les plus petites. Il a été estimé que la production régionale annuelle de bouteilles de vin s'élève à plus de 25000 et que les emplois rattachés à la culture, à la transformation et à la commercialisation dépassent un équivalent temps plein (ETP) de 50 personnes.

\section{Conditions écogéographiques particulières}

L'implantation de la vigne dans la région pourrait apparaitre comme un exploit pour une région qui frôle le $49^{\mathrm{e}}$ parallèle nord. Or, cela s'explique par des conditions écogéographiques particulières.

Le réchauffement climatique déplace vers le nord la frontière des écosystèmes réputés comme étant plus méridionaux, allonge la saison de croissance ${ }^{3}$ et apporte plus de chaleur. La climatologie fine pourrait ouvrir la voie à la découverte d'îlots plus favorables à la viticulture (Lasserre, 2017).
Des variétés de cépages hybrides plus rustiques et plus résistants au froid sont adoptées par les producteurs. Par exemple, parmi plus de 60 cépages, on compte le Vandal-Cliche, le Radisson, le Frontenac gris, l'Adalmiina, le Louise de Swenson, l'Osceola muscat, le Sainte-Croix, le Baltica, le Marquette, le Somerset ainsi que le Saint-André (un cépage indigène). Dubé et Turcotte (2011) ont produit un guide instructif et utile sur les cépages se trouvant au Québec.

Une meilleure connaissance du site en matière d'exposition au soleil (vers le sud), de terrain incliné, de bon drainage de l'eau et de l'air ainsi que de sol à texture légère permet la sélection des meilleurs endroits, voire de terroirs (Barriault, 2012).

La compréhension des facteurs qui délimitent des aires de microclimats favorables à l'agriculture permet de circonscrire les zones plus chaudes, plus ensoleillées et moins sensibles au gel en raison de la proximité de vastes étendues d'eau. À ce propos, le lac Saint-Jean et la rivière Saguenay jouent un rôle régulateur primordial.

Ajoutons à ces points l'importance d'un savoir scientifique et d'un savoir-faire basés sur une formation technique et agronomique qui s'associent à l'esprit créatif des producteurs, qui n’hésitent pas à se lancer dans l'aventure. 


\section{Intégration au paysage et à l'économie}

Comme les bleuetières, les atocatières et dernièrement les camérisières, la vigne est en train de s'ancrer dans le paysage. Plusieurs hectares supportent des formes visuelles nouvelles : des rangs allongés comme des haies au feuillage soutenu par des fils tendus, des bâtiments spécifiques rassemblant les fonctions de transformation et de dégustation ou simplement l'édification de kiosques de vente au bord de la route.

Le raisin prend le plus souvent la voie du pressoir pour finir en bouteille. Le vin produit occupe les marchés local et régional: il est consommé au vignoble, même sous forme de dégustations, et acheté sur place. On le trouve dans les marchés d'alimentation, les restaurants et les bars. Quelques vignobles s'enorgueillissent en apposant le label IGP. Certains remportent même des prix d'excellence à l'échelle internationale. Autrement, chez les plus petits propriétaires, le raisin de table est réduit à la consommation personnelle et locale ainsi qu'à l'activité de l'autocueillette.

Pour le moment, peu de vignobles font vivre son homme. C'est pourquoi des activités connexes sont pratiquées pour rentabiliser l'entreprise : la production et la vente d'alcool et de liqueurs; la culture de petits fruits, d'arbres fruitiers (pommes, prunes, griottes) et de légumes; et l'élevage).

Le développement de la vigne et des vignobles dans la région du Saguenay-Lac-Saint-Jean est tout jeune. Et ce n'est pas fini. Les projets d'agrandissement de vignobles, la naissance de vignoblesjardins communautaires, des essais de culture sous serre et des projets gouvernementaux en R-D confirment que la vocation viticole n'est pas prête à s'éteindre.

\section{Conclusion}

Les régions nordiques du Québec ne sont pas à l'écart du phénomène du réchauffement climatique. En ce qui concerne l'agriculture, les activités s'éloignent de l'image initiale affublée à la région du Saguenay-Lac-Saint-Jean, soit là où l'herbe pousse bien. On assiste à une diversification des productions et, désormais, la vigne fait non seulement partie du décor, mais aussi de la vie et de la culture régionales. L'avenir est prometteur. D'autant plus que les conditions de production en milieu froid sont mieux connues et que la détermination de nouveaux lieux de production peut s'inspirer à la fois de la réalité présente et vécue ainsi que des modèles pouvant être dérivés des systèmes d'information géographique (SIG).

\section{NOTES}

1 Cet article est un condensé de l'analyse géographique produite par l'auteur et accessible à l'adresse suivante : https://constellation.uqac.ca/7436

2 Identification géographique protégée : appellation certifiée par le Québec.

3 Selon Desorgues et Dard (2017), la saison de végétation a crû de 15 jours au cours des 30 dernières années.

\section{RÉFÉRENCES}

Barriault, É. (2012). Guide d’implantation : vigne. Centre de référence en agriculture et agroalimentaire du Québec.

Desorgues, P. et Dard, S. (2017, 25 août). Le vin du Québec, un vignoble émergent. TV 5Monde. https://information.tv5monde.com/info/le-vin-du-quebec-un-vignoble-emergent-187850

Deshaies, L. et Dubois, J.-M. (1993, 4 octobre). Vins et vignobles artisanaux au Québec. Géographes, p. 4-110.

Dubé, G. et Turcotte, I. (2011). Guide d'identification des cépages cultivés en climat froid: cépages de cuve. Richard Grenier Éditeur.

Dubois, J.-M. et Deshaies, L. (1997). Guide des vignobles du Québec : sur la route des vins. PUL/Éditions de l’IQRC.

Ferland, C. (2010). Bacchus en Canada : boissons, buveurs et ivresses en Nouvelle-France. Septentrion.

Gauthier, M.-J. (2021). Vigne et vignobles au Saguenay-Lac-Saint-Jean : étude géographique. Laboratoire d'expertise et de recherche en géographie appliquée, Groupe de recherche en études et intervention régionales, Université du Québec à Chicoutimi. https:// constellation.uqac.ca/7436 
Ingrand, S., Lurette, A., Gouttenoire, L., Sevun, J. et Moulin, C.-H. (2014). Le processus d'innovation en ferme : illustrations en élevage. INR A Productions animales, 27(2), 147-160. https://productions-animales.org/article/view/3063

Lasserre, F. (2017). Impact des changements climatiques et des conditions géographiques locales sur la maturation des cépages bybrides et rustiques au Québec [rapport final]. Département de géographie, Université Laval. https://www.ggr.ulaval.ca/sites/default/files/documents/ Lasserre/Publications/rapport_final_nov_2014_public.pdf 